\section{BRAZIULIAN JOURNAL}

OF MEDICAL AND BIOLOGICAL RESH ARCH

www.bjournal.com.br
ISSN 0100-879X

Volume 43 (01) 1-123 January 2010

BIOMEDICAL SCIENCES

AND

CLINICAL INVESTIGATION

Braz J Med Biol Res, J anuary 2010, Volume 43(1) 77-84

Brain tissue segmentation using q-entropy in multiple sclerosis magnetic resonance images

P.R.B. Diniz, L.O. Murta-Junior, D.G. Brum, D.B. de Araújo and A.C. Santos

The Brazilian Journal of Medical and Biological Research is partially financed by
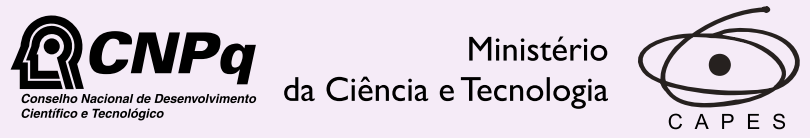

Ministério da Educação

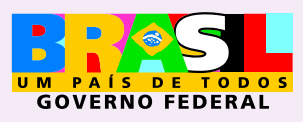

Institutional Sponsors 


\title{
Brain tissue segmentation using q-entropy in multiple sclerosis magnetic resonance images
}

\author{
P.R.B. Diniz ${ }^{1}$, L.O. Murta-Junior ${ }^{3}$, D.G. Brum ${ }^{1}$, D.B. de Araújo ${ }^{3}$ and A.C. Santos ${ }^{1,2}$ \\ ${ }^{1}$ Departamento de Neurociências e Ciências do Comportamento, ²Departamento de Clínica Médica, \\ Divisão de Radiologia, Faculdade de Medicina de Ribeirão Preto, \\ Universidade de São Paulo, Ribeirão Preto, SP, Brasil \\ ${ }^{3}$ Departamento de Física e Matemática, Faculdade de Filosofia, \\ Ciências e Letras de Ribeirão Preto, Universidade de São Paulo, Ribeirão Preto, SP, Brasil
}

\begin{abstract}
The loss of brain volume has been used as a marker of tissue destruction and can be used as an index of the progression of neurodegenerative diseases, such as multiple sclerosis. In the present study, we tested a new method for tissue segmentation based on pixel intensity threshold using generalized Tsallis entropy to determine a statistical segmentation parameter for each single class of brain tissue. We compared the performance of this method using a range of different q parameters and found a different optimal q parameter for white matter, gray matter, and cerebrospinal fluid. Our results support the conclusion that the differences in structural correlations and scale invariant similarities present in each tissue class can be accessed by generalized Tsallis entropy, obtaining the intensity limits for these tissue class separations. In order to test this method, we used it for analysis of brain magnetic resonance images of 43 patients and 10 healthy controls matched for gender and age. The values found for the entropic $q$ index were 0.2 for cerebrospinal fluid, 0.1 for white matter and 1.5 for gray matter. With this algorithm, we could detect an annual loss of $0.98 \%$ for the patients, in agreement with literature data. Thus, we can conclude that the entropy of Tsallis adds advantages to the process of automatic target segmentation of tissue classes, which had not been demonstrated previously.
\end{abstract}

Key words: Multiple sclerosis; Automatic segmentation; Magnetic resonance image; Volumetry; Tsallis entropy

\section{Introduction}

Multiple sclerosis (MS) is understood today as a genetically determined autoimmune disease, with environmental modulation, which mainly affects young adults and is characterized by multifocal and multiphasic inflammatory destruction of myelin in the central nervous system. Pathological findings include multiple areas of lymphocyte and macrophage infiltration associated with multifocal demyelination throughout the white central nervous system (1). As a disease of young people, which begins most often between 20 and 30 years of age, MS involves a very high economic and social cost because it affects people who are in their most productive phase of life (1).

There are several clinical variants of MS. The classic form is characterized by attacks, or recurrences, followed by remission, usually with complete regression of symptoms during the initial phase of the disease. However, after some time, there is a residual deficit after the attacks, followed by a gradual evolution (2). The former stage is called relapseremitting $M S(R R)$ and the latter, secondary progressive MS (SP). After a period of about 8 to 15 years, the disease usually enters the SP phase. In about $15 \%$ of patients, the disease immediately starts in the progressive phase and is called primary progressive MS (PP) (2).

The use of magnetic resonance imaging (MRI) has proved to be sensitive enough for the diagnosis and followup of the lesions and other tissue alterations with time (3). The disease burden in MS is measured by the total area of visible lesions in T2 weighted images. However, the T2 lesion volume is poorly correlated with clinical disability measured by current clinical scales (3). Stronger correlations have been found between the disability and the individual quantitative evaluation by MRI of brain tissue volumes (3).

Correspondence: L.O. Murta-Junior, Departamento de Física e Matemática, FFCLRP, USP, Av. Bandeirantes, 3900, 14040-901

Ribeirão Preto, SP, Brasil. Fax: +55-16-3602-4887. E-mail: murta@usp.br

Received May 29, 2009. Accepted October 15, 2009. Available online November 23, 2009. Published January 11, 2010. 
These findings, in agreement with histopathologic exams, suggest that permanent neurological dysfunction is associated with brain and spinal cord atrophy (4-7).

The development of atrophy seems to be associated with the progressive loss of myelin and axons and, secondarily, with neuronal apoptosis. It is apparently more evident in the late phase of the disease, when the number of lesions reaches a limit and the volumetric loss becomes perceptible.

The measurement of brain volume by MRI is a potential marker to be used to monitor the evolution of the disease. The fractional volumes of gray and white matter in MS have been investigated in a few studies (8-10). It is known that changes are not restricted to white matter, with involvement of the gray matter having also been observed (10). Moreover, recent studies have suggested that gray and white matter atrophy does not occur simultaneously, but can be related to independent degenerative processes depending on the type of disease progression.

Lukas et al. (10) have proposed the use of the brain parenchymal fraction as a marker for destructive pathological processes in patients with MS. This measurement is defined as the ratio between brain parenchymal volume and intracranial volume.

The measurements of progressive brain alterations require the use of sensitive techniques capable of detecting small variations, since temporal changes in encephalic volume are very subtle. Depending on the techniques used, brain volume reduction over a year is 0.6 to $1.0 \%$ in patients with MS. In contrast, the annual reduction of total volume of the brain in healthy controls varies from 0.1 to $0.3 \%$ and is mainly due to a decrease of gray matter (10). Although cerebral atrophies have been measured in several longitudinal studies, there is still no consensus about the most appropriate method.

\section{Material and Methods}

\section{Image acquisition and patients}

The MRI exams were acquired with a 1.5 Tesla scanner (Magneton Vision, Siemens, Germany). A standard head coil was used to acquire 40 T1-weighted 3-D axial

Table 1. Characterization of the patients studied.

\begin{tabular}{lccccc}
\hline & $\begin{array}{c}\text { Normal } \\
\text { controls }\end{array}$ & $\begin{array}{c}\text { Total No. of } \\
\text { patients }\end{array}$ & $\begin{array}{c}\text { Patients } \\
\text { with PP }\end{array}$ & $\begin{array}{c}\text { Patients } \\
\text { with RR }\end{array}$ & $\begin{array}{c}\text { Patients } \\
\text { with SP }\end{array}$ \\
\hline $\mathrm{N}$ & 10 & 43 & 4 & 27 & 12 \\
Men & 5 & 19 & 4 & 9 & 6 \\
Women & 5 & 24 & 0 & 18 & 6 \\
Age (years) & $36 \pm 11.06$ & $36.35 \pm 9.97$ & $47 \pm 11$ & $35 \pm 9.6$ & $35 \pm 9.1$ \\
\hline
\end{tabular}

Data are reported as numbers or means $\pm \mathrm{SD}$. $\mathrm{PP}=$ primary progressive multiple sclerosis; RR = relapse-remitting multiple sclerosis; $\mathrm{SP}=$ secondary progressive multiple sclerosis. slices (MPRAGE) covering the whole brain. The sequence involved the following parameters: TE, $11 \mathrm{~ms}$; TR, $34 \mathrm{~ms}$; FA, $30^{\circ}$; slice thickness, $5 \mathrm{~mm}$.

Forty-three clinically defined MS patients were selected (19 males and 24 females, mean age of 36.35 years) including the RR, SP, and PP subtypes. To obtain a higher degree of consistency, all patients were examined by a single neurologist, a specialist in demyelinating diseases, and all MRI exams were reviewed by two radiologists. Ten healthy volunteers ( 5 males and 5 females, mean age of 36 years) were studied for comparison.

Exams contaminated by artifacts, such as those caused by movement, were excluded. The study was approved by the Ethics Committee and Research of the Hospital das Clínicas of Ribeirão Preto and all patients gave written informed consent to participate in the study. Table 1 presents the demographic characteristics of the subjects, as well as patient distribution according to MS type.

\section{Algorithm for tissue volumetric quantification}

There are several studies describing brain structures observed by MRI as fractal geometry (11). The fractal dimension was found to be a sensitive marker for certain brain diseases $(12,13)$. This suggests that an entropy formulation suitable for fractal manifesting systems (14) is adequate as a segmentation marker for brain tissues.

The algorithm evaluated in the present study was developed using the NIH Image J software, which provides the automatic quantification of brain total volume, cerebrospinal fluid (CSF), white matter, and gray matter.

The segmentation method involves two stages: Snake algorithm or active contour model for encephalon extraction, and the maximum entropy algorithm for tissue segmentation. A diagram presenting the overall steps of the developed algorithm is illustrated in Figure 1.

\section{Brain segmentation}

Snake is a particular type of active contour in which an initial curve is deformed towards the border by internal and external forces (15), which can be obtained by a process of global minimization or can be considered only based on local information (15). Active contours make use of local information about a contour. The active contours are especially useful when the geometry of the object borders is unknown (15) and are an effective technique for image segmentation.

In this method, we use anisotropic filtering followed by the use of Snakes to obtain reliable encephalon segmentation. The overall effect of an anisotropic filter is a kind of smooth image and the reduction of the amount of gray level variations. This smoothing effect is controlled by the local gradient, so that the neighborhoods with gray level transitions are preserved. The controlled smoothing process tends to minimize noises, but preserves border transitions (16). 
After anisotropic filtering, a Snake algorithm was applied, with a circle as the contour for initialization, centered on the mass center of the image. Its optimum radius is heuristically defined and varies according to the image plane used. The segmentation resulting from Snake algorithm is an image with a black background and the segmented encephalon. Figure 2 shows the typical result of application of the anisotropic diffusion filter application and the Snake algorithm.

A common problem in automatic segmentation methods is the presence of non-uniformity in MRI. It appears as a smooth variation of the signal intensity across the image and is usually attributed to many factors such as poor radiofrequency (RF) field uniformity, eddy currents driven by the switching of field gradients, and patient anatomy both inside and outside the field of view (17). Although these factors contribute to 10 to $20 \%$ of variation in signal intensity, they have little impact on the visual diagnosis. However, automatic segmentation performance can be significantly reduced by this inhomogeneity (17), and such artifacts must be corrected prior to tissue classification using an inhomogeneous isotropic diffusion 2-D filter.

\section{Tissue classification}

Following the previously described pre-processing step, the encephalon extracted from MRI was segmented into different brain tissues (white matter, gray matter and CSF) by the use of an algorithm based on the maximum generalized entropy principle (18). The entropy is frequently used to indicate the amount of information produced in a certain source, and is also used to measure the disorder or complexity of a dataset.

The information entropy was conceived by Shannon intuitively rather than from basic principles (19). We can conjecture, for instance, that an event with complete certainty of occurrence, i.e., probability equal to unit, the corresponding entropy is zero. On the other hand, if an event is rare, with a very small probability of occurrence, the net entropy is large. The mathematical function of this behavior is logarithmic function. The choice of a logarithmic base in the entropy formula determines the unit for information entropy used. The most common unit of information is the bit, based on the binary logarithm. An interesting and useful property of entropy is the fact that, for a closed dynamic system, the entropy always grows to a maximum value.

This formalism has been shown to be restricted to the domain of validity of the Boltzmann-Gibbs-Shannon (BGS) statistics, and it seems to describe nature when the effective microscopic interactions and the microscopic memory are short ranged. Generally, systems that obey BGS statistics are called extensive systems. If we consider that a physical system can be decomposed into two statistical independent subsystems $A$ and $B$, the probability of the composite system is $p A+B=p A \cdot p B$. It has been shown that Shannon entropy has the following additivity property:

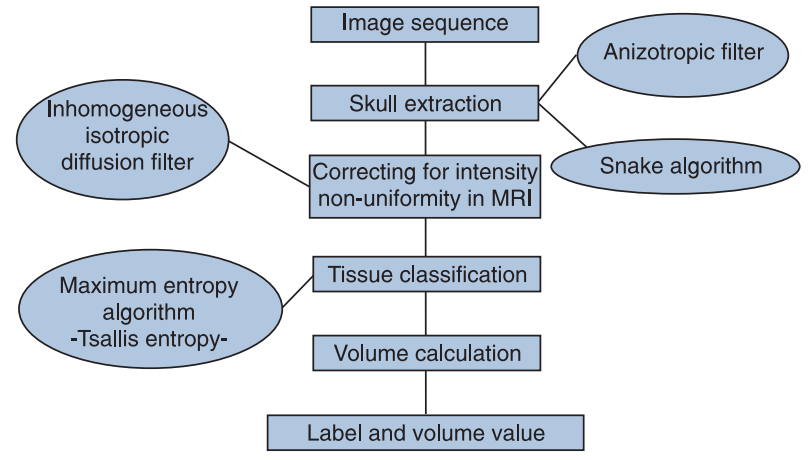

Figure 1. Diagram of the proposed algorithm. MRI = magnetic resonance image.

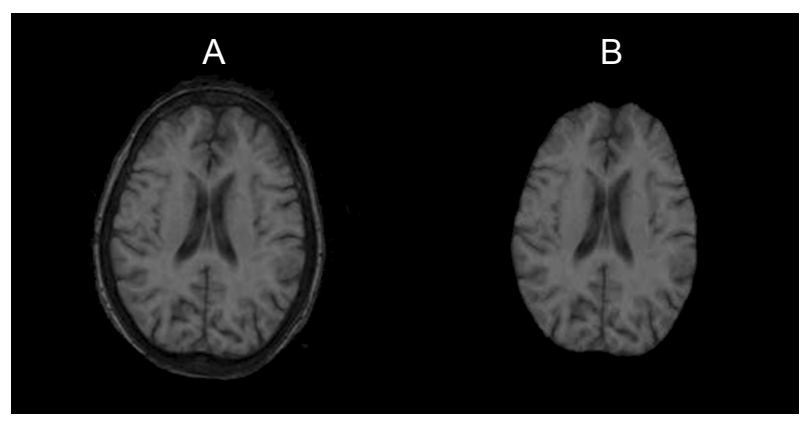

Figure 2. Illustration of the result of the first stage of the proposed method. $A$, Original magnetic resonance imaging slice; $B$, result after anisotropic filtering and active contour.

$S(A+B)=S(A)+S(B)$

However, for a certain class of physical systems, presumably those that entail long-range interactions, long-time memory and multifractal-like macrostates, an extension of this principle can be interesting (19). Inspired by multifractal concepts, Tsallis (19) has proposed a generalization of the BGS statistics, which is based on a generalized entropic form:

$S_{q}=\frac{1-\sum_{i=1}^{k}\left(p_{i}\right)^{q}}{1-q}$

where $k$ is the total number of possibilities of the system and the real number, q, is an entropic index that characterizes the degree of nonadditivity. This expression meets the BGS entropy in the $q \rightarrow 1$ limit. Tsallis entropy is nonadditive in such a way that, for a statistical independent system, the entropy of the system is defined by the following nonadditivity entropic rule:

$S_{q}(A+B)=S_{q}(A)+S_{q}(B)+(1-q) S_{q}(A) S_{q}(B)[3]$ 
Extending these concepts to the imaging context, we can consider an image as a result of a random process, with the probability $\left(p_{i}\right)$ corresponding to the probability of a certain pixel to assume a specific intensity value, i ( $i=$ $1, . ., \mathrm{G})$, that is the normalized histogram of the considered image. The intensity histogram of an image becomes the probability density function of the image just by dividing each number of pixels of intensity $\mathrm{i}, \mathrm{g}_{\mathrm{i}}$, by the number of pixels in the image, $\mathrm{N}$.

However, the entropy of an image is a positive measure and the maximum entropy occurs when an image contains the same amount of pixels for all intensities, that is, all intensities have the same probability of occurrence.

The algorithm used is based on the entropy algorithm proposed by Kapur et al. (20) and Pun (21). According to them, the entropy associated with the black pixels, $S_{b}$, and the entropy associated with the white pixels, $S_{W}$, is delimited by the threshold value $t$. The algorithm assumes that $t$ is such that it maximizes the total accumulated entropy function. $\mathrm{S}_{\mathrm{b}}$ is the accumulated entropy ranging from dark black-gray level to threshold and $S_{w}$ is the accumulated entropy from threshold to bright white-gray level. One can define $p[i]$ as the value of the probability for pixels in a particular gray level. In the present context, the q parameter represents how a particular tissue accumulates information in the images, according to the generalized statistics. Thus, as can be seen in Equation 3, q values tell us the way system incorporates entropy or information from subsystems.

The first structure segmented by this process is the CSF, which is subtracted from the original image. The image without the CSF is submitted again to the algorithm of maximum entropy and gray matter is segmented. Once the gray matter is subtracted, one ends with the white matter. Figure 3 shows an example of segmentation based on this technique. In the MRI context, the gray level probability distribution is directly correlated with the overall fractal constituent tissue structure. Therefore, if one considers

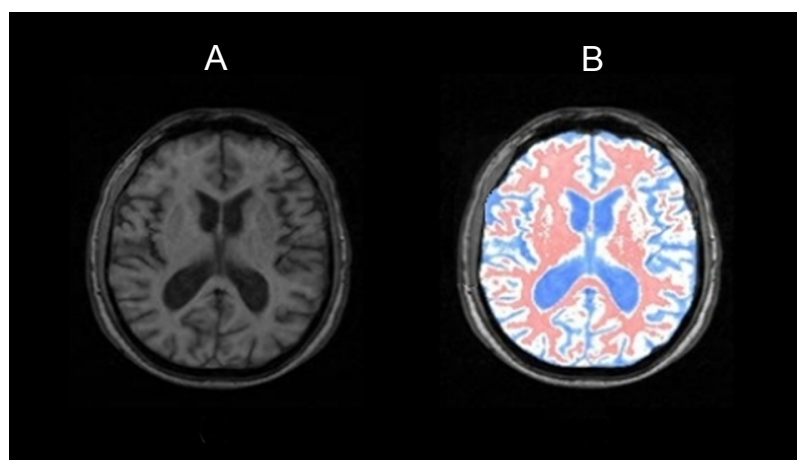

Figure 3. Maximum entropy segmentation example. $A$, Original image; $B$, image with the segmentation masks. Blue indicates cerebrospinal fluid, white indicates the gray matter, and red indicates the white matter. different overall structure images having different statistical distributions, this will lead to a different threshold that corresponds to the maximum parametric entropy principle.

\section{Quantitative evaluation of the segmentation results}

Segmentation results were compared with the manually segmented volume as delineated by a specialist. This comparison was made using the following error types:

Total error. The total error, denoted by " $\varepsilon \mathrm{T}$ ", is calculated as:

$\varepsilon_{T}=\frac{|V(A)-V(M)|}{V(M)} \times 100 \%$

where $A$ is the segmentation by the proposed algorithm, $M$ is the manual segmentation performed by the specialist, and $\mathrm{V}(\mathrm{A})$ and $\mathrm{V}(\mathrm{M})$ are the respective volumes of the segmentation.

False-positive error. A false-positive (FP) voxel is a voxel that is selected in automatic algorithm segmentation but not in the manually segmented volume. The FP error, denoted by " $\varepsilon_{\mathrm{FP}}$ ", is calculated as:

$\varepsilon_{F P}=\frac{V(A)-V(A \cap M)}{V(M)} \times 100 \%$

False-negative error. A false-negative (FN) voxel is the voxel selected by manual segmentation but not by automatic algorithm segmentation. The FN error, denoted by " $\varepsilon_{\mathrm{FN}}$ ", is calculated as:

$\varepsilon_{F N}=\frac{V(M)-V(A \cap M)}{V(M)} \times 100 \%$

\section{Accuracy tests with simulated MRI}

We used simulated MRI data (BrainWeb, http://www. bic.mni.mcgill.ca/brainweb/) (22) to evaluate the accuracy of segmentation in terms of similarity by comparing our segmented tissue masks to the gold standard tissue masks. The segmentation algorithm was evaluated for the effects of radio frequency inhomogeneities (at 0, 20, and 40\%) and noise $(0,1,3,5,7$, and $9 \%)$ using the similarity index (Si) (23). This index is calculated as:

$S_{i}=\frac{2 \times T P}{2 \times T P+F P+F N}$

where TP (correctly labeled), FP and FN are, respectively, the number of true-positive, false-positive and false-negative voxels. The parameter used for evaluation indicates that a similarity index of 0.7 or above is considered to indicate good agreement.

\section{Brain parenchymal fraction}

Atrophy is calculated by the brain parenchymal fraction (BPF), which is expressed by the percentage of intracranial volume (ICV) occupied by the brain, where the ICV 


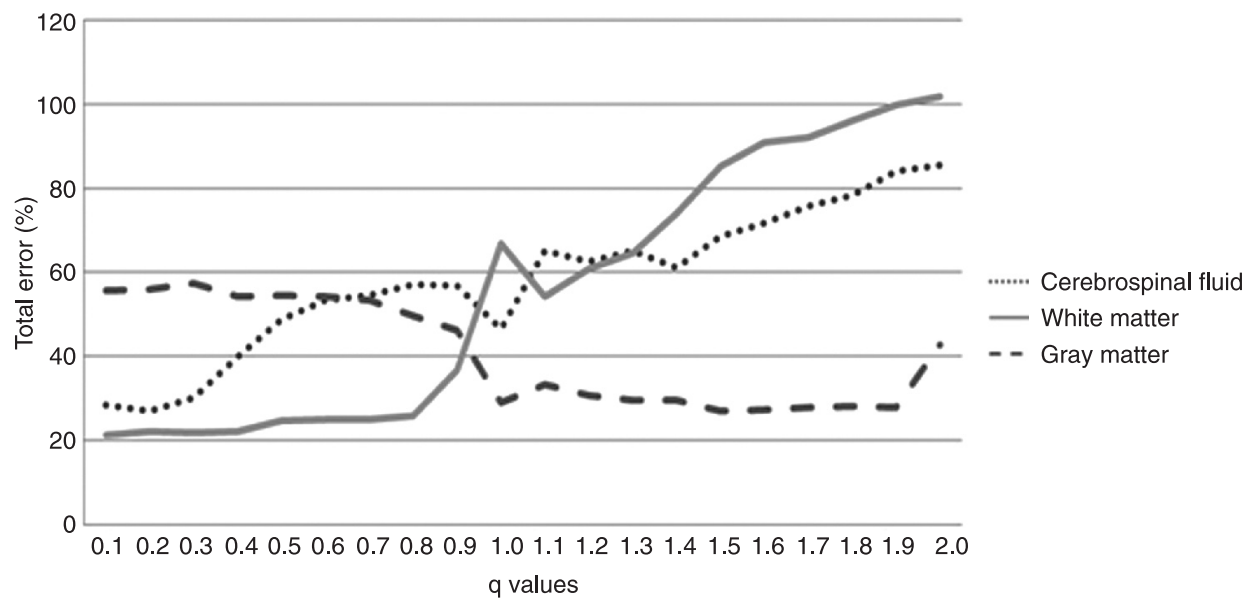

Figure 4. Relative error for each segmented tissue as a function of $q$ value.

Table 2. Calculated tissue q value.

\begin{tabular}{lcc}
\hline Tissue & q value & Total error \\
\hline Cerebrospinal fluid & 0.2 & $27 \%$ \\
White matter & 0.1 & $21 \%$ \\
Gray matter & 1.5 & $27 \%$ \\
\hline
\end{tabular}

The $\mathrm{q}$ values were determined by minimizing the relative errors for each tissue.

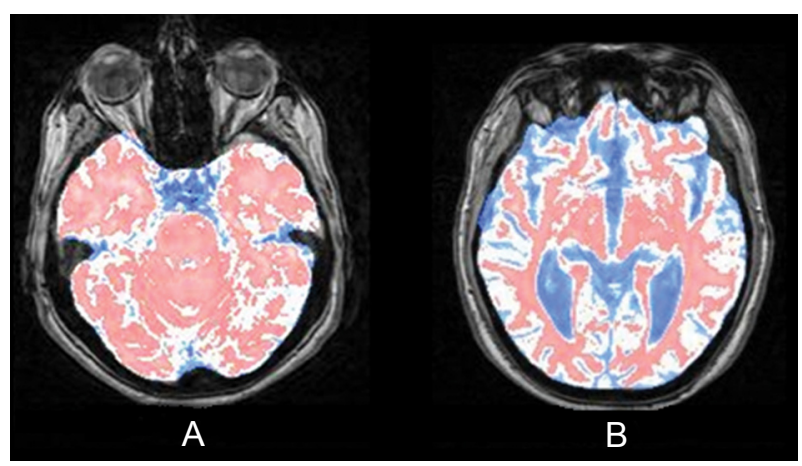

Figure 5. Result obtained with the proposed tool based on a deformable model (Snake). $A$, Image of skull base. $B$, Image of eye region.

is defined as the sum of CSF + white matter (WM) + gray matter (GM). BPF is then calculated by the sum of WM + GM divided by the ICV, multiplied by 100 , yielding the value in percentage. This formula is presented below:

$B P F=\frac{W M+G M}{W M+G M+C S F} \times 100 \%$

\section{Results}

\section{Searching for the best parametric q value for tissue segmentation}

The entropic index, q, can be interpreted as a parameter that characterizes the degree of non-extensivity of a system. In image segmentation, the non-extensivity of a system can be interpreted as the presence of correlations among pixels of the same object in the image. These correlations can be classified as long-range correlations in the case of images that present pixels strongly correlated in terms of gray levels and scale invariant spatial filling.

In the development of this method, we used positive values of $q$ to search for the optimal value from 0.1 to 2.0 and investigated the threshold level for visual inspection and for the smallest relative error of the volume for all structures.

The error analysis showed that the best $q$ values for CSF, white matter and gray matter are 0.2, 0.1 and 1.5, respectively. The graph in Figure 4 and Table 2 summarize the results of the experiment.

\section{Total volume evaluation}

Figure 5 shows the result of extraction of the brain tissue in two images, one located at the base of the skull and the other in the region of the eyes. The region of the eyes is of difficult limitation since it presents signal intensity values similar to those of brain tissue. Traces of the occipital and temporal lobes appear at the skull base, whose respective limits are well defined; the difficulty is to delimit them as a single structure. It is important to observe a good performance of the developed tool regarding the eyeball region. A nonsignificant $(P \geq 0.05)$ difference of $21.75 \mathrm{~cm}^{3}$ in time can be observed.

\section{Cerebrospinal fluid, white matter and gray matter segmentation}

Using BrainWeb-simulated MR images, the similarity 
Table 3. Similarity indices at various radiofrequency (RF) inhomogeneity and noise levels in an accuracy test using BrainWeb simulation images.

\begin{tabular}{|c|c|c|c|}
\hline \multirow[t]{2}{*}{ Noise level } & \multicolumn{3}{|c|}{ RF inhomogeneity levels } \\
\hline & $0 \%$ & $20 \%$ & $40 \%$ \\
\hline \multicolumn{4}{|l|}{ Gray matter } \\
\hline $0 \%$ & 0.86903 & 0.797637 & 0.804889 \\
\hline $1 \%$ & 0.817627 & 0.816415 & 0.820566 \\
\hline $3 \%$ & 0.832996 & 0.832575 & 0.830091 \\
\hline $5 \%$ & 0.808635 & 0.806584 & 0.808027 \\
\hline $7 \%$ & 0.762137 & 0.767459 & 0.769204 \\
\hline $9 \%$ & 0.715715 & 0.721006 & 0.720388 \\
\hline \multicolumn{4}{|c|}{ White matter } \\
\hline $0 \%$ & 0.85809 & 0.856086 & 0.859677 \\
\hline $1 \%$ & 0.866448 & 0.863903 & 0.864305 \\
\hline $3 \%$ & 0.876857 & 0.875415 & 0.872875 \\
\hline $5 \%$ & 0.863628 & 0.860913 & 0.861685 \\
\hline $7 \%$ & 0.835257 & 0.839319 & 0.838922 \\
\hline $9 \%$ & 0.80627 & 0.812064 & 0.812882 \\
\hline \multicolumn{4}{|c|}{ Cerebrospinal fluid } \\
\hline $0 \%$ & 0.767662 & 0.769236 & 0.772654 \\
\hline $1 \%$ & 0.789707 & 0.797884 & 0.808227 \\
\hline $3 \%$ & 0.816233 & 0.82367 & 0.827553 \\
\hline $5 \%$ & 0.80185 & 0.80318 & 0.805297 \\
\hline $7 \%$ & 0.775803 & 0.777564 & 0.777821 \\
\hline $9 \%$ & 0.738604 & 0.740258 & 0.741042 \\
\hline
\end{tabular}

index (22) for GM, WM and CSF mask images was 0.832, 0.875 , and 0.823 at $3 \%$ noise and $20 \%$ RF inhomogeneity level. A similarity index of 0.7 or above is considered to indicate good agreement (22). The segmentation method presented here works well in the presence of RF inhomogeneity and the various noise levels, as can be seen in Table 3.

Volume, manual segmentation and automatic segmentation values were compared by the Student $t$-test for paired samples and the total error, false-positive error and false-negative error were determined.

The results showed that there was no significant difference between the volumes measured by manual segmentation and automatic segmentation for the CSF (347.22 \pm 94.07 vs $322.842 \pm 114.43 \mathrm{~cm}^{3}$, respectively). The mean relative error was $27 \%$ with an $\varepsilon_{\mathrm{FN}}$ of $15 \%$ and an $\varepsilon_{\mathrm{FP}}$ of $11 \%$.
Table 4. Errors by region of interest.

\begin{tabular}{lccc}
\hline & Total error & FN error & FP error \\
\hline Cerebrospinal fluid & $27 \%$ & $15 \%$ & $11 \%$ \\
White matter & $21 \%$ & $8 \%$ & $14 \%$ \\
Gray matter & $27 \%$ & $14 \%$ & $12 \%$ \\
\hline
\end{tabular}

$\mathrm{FN}=$ false-negative; $\mathrm{FP}=$ false-positive

Table 5. Annual measured volume loss as a function of clinical classification.

\begin{tabular}{lcccc}
\hline & Total & PP & RR & SP \\
\hline $\mathrm{N}$ & 43 & 4 & 27 & 12 \\
Age (years) & $36.35 \pm 9.97$ & $47 \pm 11$ & $35 \pm 9.6$ & $35 \pm 9.1$ \\
Annual loss & $0.98 \%$ & $1.15 \%$ & $1.01 \%$ & $0.66 \%$ \\
\hline
\end{tabular}

Data are reported as means $\pm \mathrm{SD}$. PP = primary progressive multiple sclerosis; $\mathrm{RR}=$ relapse-remitting multiple sclerosis; $\mathrm{SP}=$ secondary progressive multiple sclerosis.

For the white matter volumes, the test showed no significant differences between the values obtained by manual segmentation and automatic segmentation using the method presented here ( $556.218 \pm 120.86$ vs 574.44 $\pm 166.97 \mathrm{~cm}^{3}$, respectively). The relative error found was $21 \%$ with an $\varepsilon_{\mathrm{FN}}$ of $8 \%$ and an $\varepsilon_{\mathrm{FP}}$ of $14 \%$.

The Student $t$-test for paired samples showed no significant differences between the gray matter volumes obtained by manual segmentation and by automatic segmentation (459.01 \pm 145.26 vs $412.72 \pm 106.27 \mathrm{~cm}^{3}$, respectively). The relative error found was $27 \%$ with an $\varepsilon_{\mathrm{FN}}$ of $14 \%$ and an $\varepsilon_{\mathrm{FP}}$ of $12 \%$. Table 4 shows these results.

\section{Brain parenchymal fraction}

It is important to note that the patients were compared to control individuals matched for age and gender. The influence of age on brain atrophy is known. The BPF was estimated at $76.23 \pm 5.75 \%$ for the controls and at 74.12 $\pm 4.23 \%$ for the patients. The annual loss was calculated by the clinical classification of the patients. In general, MS patients presented an annual loss of $0.98 \%$. For each clinical classification, we found an annual volumetric loss of $1.15 \%$ for patients in the PP phase, of $1.01 \%$ for patients in the RR phase and of $0.66 \%$ for patients in the SP phase. Table 5 presents these results.

The amount of atrophy is obtained from the BPF, calculated from the volumetric data acquisition as the percentile rate of intracranial volume occupied by the encephalon, which is the sum of CSF, WM and GM. 


\section{Discussion}

Brain tissue segmentation is the key image processing tool allowing a precise quantification of atrophy in degenerative diseases such as MS. Brain volume quantification when performed manually, although a simple procedure, is time consuming and subject to wide variability between different experts. Alternatively, one can use automated segmentation procedures. The algorithm to be used should be precise enough to detect small differences and robust enough to allow reproducible measurements to be used in evolutionary evaluations.

The intensity threshold segmentation technique has not yet been used successfully due to the lack of a reliable automatic method (24-26). Therefore, we propose here a segmentation method based on the search for the intensity threshold by maximizing generalized Tsallis entropy (19). Generalized Tsallis entropy is a formulation for statistical mechanic entropy that considers the long-range correlations between system bodies and multifractal phase space (14). While classical Shannon entropy considers the particles of a system to be statistically independent, Tsallis entropy considers their interdependence. These concepts can be transported into the context of image processing in view of the correlation between differently spaced pixels and the fractal pattern or scale invariant similarity observed in certain images.

Within the context of neuroimaging studies, we have already used Tsallis entropy applied to functional MRI $(27,28)$. Tsallis generalized entropy was used in the current study in order to optimize the segmentation threshold. In the Tsallis formulation, there is a q parameter related to the statistical consequences of pixel correlations. Deviation of q from unit is a measure of the departure from regular geometries, or from random statistics.

When compared to classical Shannon entropy, this approach seems to be much more suitable for tissue segmentation. Figure 6 shows an example of this segmentation. Parametric Tsallis entropy can separate the different structures using different q values. These results suggest that each brain tissue has its own fractal-like geometry.

The clinical application of these atrophy calculation techniques is important, mainly for the evaluation of neural degenerative disease progress, and of patient response to specific treatments. When made manually, this measurement is time consuming and requires specific training. The use of tools that make this task easier is of key importance in clinical practice.

The automatic segmentation method proposed here proved to be very practical and reproducible, with small variability, for the quantitation of CSF, WM and GM using

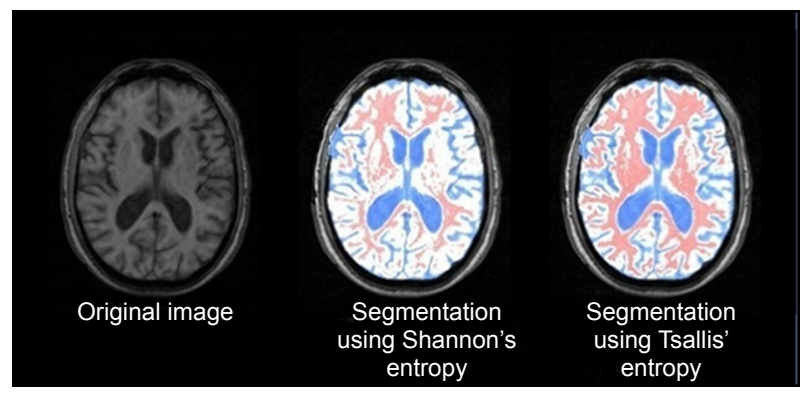

Figure 6. Segmentation using Shannon and Tsallis entropies.

the whole intracranial volume, and for the calculation of BPF using the sum of WM and GM.

Our results showed a more intense atrophy in patients considered as a whole compared with the control group. When the patients were divided into subgroups according to disease stage, atrophy became significantly more intense in patients in the PP phase, followed by those in the RR and $\mathrm{SP}$ phases. Comparison of atrophy data with the clinical data of the patients yielded encouraging results, with the patterns of atrophy detected by the algorithm being consistent with the phase of MS clinically determined in the patients. It is important to note that the average volume loss of $0.98 \%$ for the group as a whole, $1.15 \%$ for those classified as PP, $1.01 \%$ for those classified as RR, and $0.66 \%$ for patients SP agreed with the rates reported by Lukas et al. (10), which were 0.6 to $1.0 \%$ in MS patients.

The use of Tsallis entropy produces better results in the targeting of tissues to obtain a better identification of the threshold and the inclusion of partial volume between classes. The ideal q values for the segmentation of the classes are: $\mathrm{CSF}=0.2, \mathrm{WM}=0.1, \mathrm{GM}=1.5$, which have not been shown previously.

The algorithm described here is very fast $(20 \mathrm{~s}$ for segmentation) and it worked well even with thick slice in the presence of RF inhomogeneity and at the various noise levels. These characteristics allow its application to clinical routine.

It resulted in a computer algorithm capable of detecting volume loss in patients with progressive stages of the disease, distinguishing the values for patients in the early stages of multiple sclerosis from control values.

\section{Acknowledgments}

Research partially supported by CAPES, FAPESP and CNPq. 


\section{References}

1. McFarland HF. The lesion in multiple sclerosis: clinical, pathological, and magnetic resonance imaging considerations. $J$ Neurol Neurosurg Psychiatry 1998; 64 (Suppl 1): S26-S30.

2. Lublin FD, Reingold SC. Defining the clinical course of multiple sclerosis: results of an international survey. National Multiple Sclerosis Society (USA) Advisory Committee on Clinical Trials of New Agents in Multiple Sclerosis. Neurology 1996; 46: 907-911.

3. Rovaris M, Filippi M. Magnetic resonance techniques to monitor disease evolution and treatment trial outcomes in multiple sclerosis. Curr Opin Neurol 1999; 12: 337-344.

4. Losseff NA, Webb SL, O'Riordan JI, Page R, Wang L, Barker GJ, et al. Spinal cord atrophy and disability in multiple sclerosis. A new reproducible and sensitive MRI method with potential to monitor disease progression. Brain 1996; 119 (Part 3): 701-708.

5. Phillips MD, Grossman RI, Miki Y, Wei L, Kolson DL, van Buchem MA, et al. Comparison of T2 lesion volume and magnetization transfer ratio histogram analysis and of atrophy and measures of lesion burden in patients with multiple sclerosis. AJNR Am J Neuroradiol 1998; 19: 1055-1060.

6. Edwards SG, Gong QY, Liu C, Zvartau ME, Jaspan T, Roberts $\mathrm{N}$, et al. Infratentorial atrophy on magnetic resonance imaging and disability in multiple sclerosis. Brain 1999; 122 (Part 2): 291-301.

7. Liu C, Edwards S, Gong Q, Roberts N, Blumhardt LD. Three dimensional MRI estimates of brain and spinal cord atrophy in multiple sclerosis. J Neurol Neurosurg Psychiatry 1999; 66: 323-330.

8. Ge Y, Grossman RI, Udupa JK, Babb JS, Nyul LG, Kolson $\mathrm{DL}$. Brain atrophy in relapsing-remitting multiple sclerosis: fractional volumetric analysis of gray matter and white matter. Radiology 2001; 220: 606-610.

9. Chard DT, Griffin CM, Parker GJ, Kapoor R, Thompson AJ, Miller $\mathrm{DH}$. Brain atrophy in clinically early relapsing-remitting multiple sclerosis. Brain 2002; 125: 327-337.

10. Lukas C, Hahn HK, Bellenberg B, Rexilius J, Schmid G, Schimrigk SK, et al. Sensitivity and reproducibility of a new fast 3D segmentation technique for clinical MR-based brain volumetry in multiple sclerosis. Neuroradiology 2004; 46: 906-915.

11. Kiselev VG, Hahn KR, Auer DP. Is the brain cortex a fractal? Neuroimage 2003; 20: 1765-1774.

12. Esteban FJ, Sepulcre J, de Mendizaba NV, Goni J, Navas $\mathrm{J}$, de Miras Jr, et al. Fractal dimension and white matter changes in multiple sclerosis. Neuroimage 2007; 36: 543549.

13. Ha TH, Yoon U, Lee KJ, Shin YW, Lee JM, Kim IY, et al. Fractal dimension of cerebral cortical surface in schizophrenia and obsessive-compulsive disorder. Neurosci Lett 2005;
384: $172-176$

14. Lyra ML, Tsallis C. Nonextensivity and multifractality in lowdimensional dissipative systems. Phys Rev Letters 1998; 80: 53-56.

15. Kass M, Witkin A, Terzopoulos D. Snakes: Active contour models. Int J Comp Vision 1988; 1: 321-331.

16. Perona P, Malik J. Scale-space and edge detection using anisotropic diffusion. IEEE Trans Pattern Anal Mach Intell 1990; 2: 10.

17. Sled JG, Zijdenbos AP, Evans AC. A nonparametric method for automatic correction of intensity nonuniformity in MRI data. IEEE Trans Med Imaging 1998; 17: 87-97.

18. Portes de Albuquerque M, Esquef IA, Gesualdi Mello AR, Portes de Albuquerque M. Image thresholding using Tsallis entropy. Pattern Recognit Lett 2004; 25: 1059-1065.

19. Tsallis C. Possible generalization of Boltzmann-Gibbs statistics. J Stat Phys 1988; 52: 8.

20. Kapur JN, Sahoo PK, Wong KC. A new method for gray-level picture thresholding using the entropy of the histogram. Comput Vis Graphics Image Process 1985; 29: 273-285.

21. Pun T. A new method for gray-level picture thresholding using the entropy of the histogram. Signal Process 1980; 2 : 223-237.

22. Collins DL, Zijdenbos AP, Kollokian V, Sled JG, Kabani NJ, Holmes CJ, et al. Design and construction of a realistic digital brain phantom. IEEE Trans Med Imaging 1998; 17: 463-468.

23. Zijdenbos AP, Dawant BM, Margolin RA, Palmer AC. Morphometric analysis of white matter lesions in MR images: method and validation. IEEE Trans Med Imaging 1994; 13 : 716-724.

24. Nakib A, Oulhadj H, Siarry P. Fractional differentiation and non-Pareto multiobjective optimization for image thresholding. Eng Appl Artif Intell 2009; 22: 236-249.

25. Nakib A, Cooren Y, Oulhadj H, Siarry P. Magnetic resonance image segmentation based on two-dimensional exponential entropy and a parameter free PSO. In: Anonymous, Artificial Evolution. Berlin/Heidelberg: 2008. p 50-61.

26. El-Fegh I, Galhoud M, Sid-Aadhmed MA, Ahmadi M. Threelevel gray-scale images segmentation using non-extensive entropy. In: Anonymous, Computer graphics, imaging and visualisation. CGIV '07; 2007: 304-307.

27. de Araujo DB, Tedeschi W, Santos AC, Elias J Jr, Neves UP, Baffa O. Shannon entropy applied to the analysis of eventrelated fMRI time series. Neuroimage 2003; 20: 311-317.

28. Tedeschi W, Müller HP, de Araujo DB, Santons AC, Neves UPC, Ernè SN, et al. Generalized mutual information tests applied to fMRI analysis. Physica A: Statistical Mechanics and its Applications 2005; 352: 629-644. 